Marquette University

e-Publications@Marquette

Mathematics, Statistics and Computer Science

Mathematics, Statistics and Computer Science,

Faculty Research and Publications

Department of

$1-1-2010$

\title{
Character Degrees of Normally Monomial Maximal Class 5-Groups
}

Michael Slattery

Marquette University, michael.slattery@marquette.edu

Published version. Contemporary Mathematics, Volume 524 (2010), Publisher website. (C) 2010 American Mathematical Society. Used with permission.

First published in Contemporary Mathematics in Volume 524, 2010, published by the American Mathematical Society. 


\title{
Character Degrees of Normally Monomial Maximal Class 5-Groups
}

\author{
Michael C. Slattery
}

\begin{abstract}
This paper will impose limits on the possible sets of irreducible character degrees of a normally monomial 5-group of maximal class.
\end{abstract}

\section{Introduction}

Let $G$ be a finite $p$-group. Then $G$ is an M-group ("monomial") which means that every irreducible ordinary character of $G$ can be induced from a linear character of some subgroup. If one can always choose the subgroup from which one is inducing the linear character to be normal, then we say $G$ is an nM-group ("normally monomial"). Recently some papers $([3],[6])$ have studied the character degrees of normally monomial $p$-groups and especially, normally monomial $p$-groups of maximal class.

In this paper we will prove:

THEOREM. Let $G$ be a normally monomial, maximal class 5-group. Then $\operatorname{cd}(G)$ is either $\left\{1,5,25,5^{4}\right\}$, the set of all powers of 5 up to some limit, $\left\{1,5,25, \ldots, 5^{k}\right\}$ with $k \geq 1$, or either of those two forms with degree 25 removed.

Throughout the paper, the computer algebra system Magma [2] was used to gain insight, verify computations, and compute required small cases.

REMARK. In computations of character degrees of all maximal class 5-groups of order up to $5^{13}$ and some up to $5^{15}$, no groups have been found with character degrees of the form $\left\{1,5,25,5^{4}\right\}$ or $\left\{1,5,5^{4}\right\}$. Furthermore, all these groups have character degrees $\left\{1,5,5^{3}\right\}$ or $\left\{1,5,25, \ldots, 5^{k}\right\}$ even when having nilpotence class of $P_{1}$ greater than 2 or $G$ not normally monomial.

\section{Certain Module Homomorphisms}

In this paper, we are only concerned with $p=5$. Nonetheless, we will occasionally use " $p$ " for " 5 " in order to make certain formulas more readable or familiar.

We need to set up the machinery from $[5$, Section 8.2]. Let $K$ be the 5 th local cyclotomic number field and $\mathcal{O}$ be the ring of integers in $K$.

2000 Mathematics Subject Classification. $20 \mathrm{C} 15$.

(C)2010 American Mathematical Society 
In $\mathcal{O}$, let $\theta$ be a fixed primitive 5 th root of unity. Note that multiplication by $\theta$ is an additive automorphism of $\mathcal{O}$ which has order 5 . Thus we can use this action to view $\mathcal{O}$ as a $C_{5}$-module.

Let $\kappa=\theta-1$ and $\mathfrak{p}=(\kappa)$. Then $\mathfrak{p}$ is the unique maximal ideal of $\mathcal{O}$, $\left|\mathfrak{p}^{i}: \mathfrak{p}^{i+1}\right|=p$ for all $i \geq 1$, and $\mathfrak{p}^{p-1}=(p)$.

DEFinition 2.1. For any $\zeta \in \mathcal{O}$, define the $\kappa$-weight of $\zeta$ to be the smallest positive integer $i$ such that $\zeta \notin \mathbf{p}^{i}$. For instance, units of $\mathcal{O}$ have $\kappa$-weight 1 .

The patterns of commutators in our groups will be closely related to homomorphisms from $\mathcal{O} \wedge \mathcal{O}$ to $\mathcal{O}$, so we wish to examine some of these maps. For any integer $a$ coprime to 5 , define $\sigma_{a}$ to be a ring automorphism of $\mathcal{O}$ which maps $\theta$ to $\theta^{a}$. We then define $S_{2}: \mathcal{O} \wedge \mathcal{O} \rightarrow \mathcal{O}$ by

$$
(x \wedge y) S_{2}=\left(x \sigma_{2}\right)\left(y \sigma_{-1}\right)-\left(y \sigma_{2}\right)\left(x \sigma_{-1}\right)
$$

We would also like to define $\kappa_{a}=\kappa \sigma_{a}$ and $u_{a}$ to be the unit in $\mathcal{O}$ such that $\kappa_{a}=\kappa u_{a}$.

LEMMA 2.2. The value $\left(\kappa^{j+1} \wedge \kappa^{j}\right) S_{2}$ has $\kappa$-weight $2 j+2, j \geq 0$.

Proof. We compute

$$
\begin{aligned}
\left(\kappa^{j+1} \wedge \kappa^{j}\right) S_{2} & =\kappa_{2}^{j+1} \kappa_{-1}^{j}-\kappa_{2}^{j} \kappa_{-1}^{j+1} \\
& =\kappa^{2 j+1}\left(u_{2}^{j+1} u_{-1}^{j}-u_{2}^{j} u_{-1}^{j+1}\right) \\
& =\kappa^{2 j+1}\left(u_{2}-u_{-1}\right)\left(u_{2} u_{-1}\right)^{j} \\
& \in \mathfrak{p}^{2 j+1} \backslash \mathfrak{p}^{2 j+2}
\end{aligned}
$$

where the last step is true because both $\left(u_{2}-u_{-1}\right)$ and clearly $\left(u_{2} u_{-1}\right)^{j}$ are units. Therefore $\left(\kappa^{j+1} \wedge \kappa^{j}\right) S_{2}$ has $\kappa$-weight $2 j+2$.

Similarly, we have

LEMMA 2.3. The value $\left(\kappa^{j+2} \wedge \kappa^{j}\right) S_{2}$ has $\kappa$-weight $2 j+3, j \geq 0$.

Proof. As above, using the fact that $\left(u_{2}^{2}-u_{-1}^{2}\right)$ is a unit in $\mathcal{O}$.

Just to simplify notation, we write $T_{1}$ for the map $\kappa^{-1} S_{2}$ (Note: This is not precisely the map $T_{1}$ in $[5$, p.162]. It differs by a unit multiple).

COROLLARY 2.4. The value $\left(\kappa^{j+1} \wedge \kappa^{j}\right) T_{1}$ has $\kappa$-weight $2 j+1$ and $\left(\kappa^{j+2} \wedge \kappa^{j}\right) T_{1}$ has $\kappa$-weight $2 j+2, j \geq 0$.

In order to define another homomorphism, we need more detailed information about $\mathcal{O} \wedge \mathcal{O}$. Let $\mathbb{Z}_{5}$ denote the 5-adic integers. Then we can view $\mathcal{O}$ as a free $\mathbb{Z}_{5}$-module of rank 4 generated by $1, \theta, \theta^{2}$ and $\theta^{3}$. With this view, it is clear that $\mathcal{O} \wedge \mathcal{O}$ is a free $\mathbb{Z}_{5}$-module of rank 6 generated by

$$
\theta \wedge 1, \theta^{2} \wedge 1, \theta^{2} \wedge \theta, \theta^{3} \wedge 1, \theta^{3} \wedge \theta, \theta^{3} \wedge \theta^{2}
$$

On the other hand, by Proposition 8.3.5 of [5], $\mathcal{O} \wedge \mathcal{O}$ is the direct sum of a free $\mathbb{Z}_{5} C_{5}$-module of rank 1 generated by $\kappa \wedge 1$ and a free $\mathbb{Z}_{5}$-module generated by an element $z$ satisfying certain conditions. In the case of $p=5$, the element $z=\theta \wedge 1+\theta^{3} \wedge 1+\theta^{3} \wedge \theta^{2}$ meets the conditions. Therefore $\mathcal{O} \wedge \mathcal{O}$ is also generated over $\mathbb{Z}_{5}$ by

$$
(\kappa \wedge 1),(\kappa \wedge 1) \theta,(\kappa \wedge 1) \theta^{2},(\kappa \wedge 1) \theta^{3},(\kappa \wedge 1) \theta^{4}, z
$$

In order to convert from one basis to another, we can expand each element in $\mathrm{B} 2$ in terms of B1. For instance,

$$
\kappa \wedge 1=(\theta-1) \wedge 1=(\theta \wedge 1)-(1 \wedge 1)=\theta \wedge 1
$$

and, using the diagonal action of $C_{5}$ on $\mathcal{O} \wedge \mathcal{O}$

$$
\begin{aligned}
(\kappa \wedge 1) \theta^{3} & =\left(\kappa \theta^{3}\right) \wedge\left(\theta^{3}\right) \\
& =\left(\theta^{4}-\theta^{3}\right) \wedge \theta^{3}=\theta^{4} \wedge \theta^{3} \\
& =\left(\left(-\theta^{3}-\theta^{2}-\theta-1\right) \wedge \theta^{3}\right. \\
& =-\left(\theta^{2} \wedge \theta^{3}\right)-\left(\theta \wedge \theta^{3}\right)-\left(1 \wedge \theta^{3}\right) \\
& =\left(\theta^{3} \wedge 1\right)+\left(\theta^{3} \wedge \theta\right)+\left(\theta^{3} \wedge \theta^{2}\right)
\end{aligned}
$$

and so on. This produces the translation matrix $W$

\begin{tabular}{c|ccccccc} 
& $\theta \wedge 1$ & $\theta^{2} \wedge 1$ & $\theta^{2} \wedge \theta$ & $\theta^{3} \wedge 1$ & $\theta^{3} \wedge \theta$ & $\theta^{3} \wedge \theta^{2}$ \\
\hline$\kappa \wedge 1$ & 1 & 0 & 0 & 0 & 0 & 0 \\
$(\kappa \wedge 1) \theta$ & 0 & 0 & 1 & 0 & 0 & 0 \\
$(\kappa \wedge 1) \theta^{2}$ & 0 & 0 & 0 & 0 & 0 & 1 \\
$(\kappa \wedge 1) \theta^{3}$ & 0 & 0 & 0 & 1 & 1 & 1 \\
$(\kappa \wedge 1) \theta^{4}$ & 1 & 1 & 0 & 1 & 0 & 0 \\
$z$ & 1 & 0 & 0 & 1 & 0 & 1
\end{tabular}

We can now define a homomorphism $T^{*} \in \operatorname{Hom}_{C_{p}}\left(\wedge^{2} \mathcal{O}, \mathcal{O} / \mathfrak{p}^{n-m}\right)$ where $n>m \geq 4$ are integers which will be specified later. For now, $n-m$ can be viewed as an arbitrary positive integer. Our map will be defined using the generators B2 above. In particular.

$$
(\kappa \wedge 1) T^{*}=\mathfrak{p}^{n-m}, \quad z T^{*}=\kappa^{n-m-1}+\mathfrak{p}^{n-m} .
$$

LEMMA 2.5. The values $\left(\kappa^{2} \wedge 1\right) T^{*},\left(\kappa^{2} \wedge \kappa\right) T^{*}$, and $\left(\kappa^{3} \wedge 1\right) T^{*}$ all have $\kappa$-weight $n-m$ and $\left(\kappa^{i} \wedge \kappa^{j}\right) T^{*}=\mathfrak{p}^{n-m}$ for all other values of $i>j \geq 0$.

Proof. The value of $(x) T^{*}$ is determined by the coefficient of $z$ in $x$ relative to the basis B2 above. If that coefficient is a multiple of 5 , then, since $(5)=\mathfrak{p}^{4}=\left(\kappa^{4}\right)$, the $\kappa$-weight of $(x) T^{*}$ will be at least $4+$ the $\kappa$-weight of $(z) T^{*}$. That is $4+n-m$ and so, in the quotient module, $(x) T^{*}=\mathfrak{p}^{n-m}$. However, if $i$ or $j$ is at least 4, we can factor out a scalar value of 5 showing that the coefficient of $z$ must be a multiple of 5 . Hence, $\left(\kappa^{i} \wedge \kappa^{j}\right) T^{*}=\mathfrak{p}^{n-m}$ for $i>j \geq 4$. To finish the proof, it suffices to compute the $z$ component of $\kappa^{i} \wedge \kappa^{j}$ for $3 \geq i>j \geq 0$. In each case, we can expand compen thanslation matrix $W$ to switch to basis B2. In that way we find

to switch to basis
Coefficient of $z$

$$
\begin{array}{c|ccc}
\wedge & 1 & \kappa & \kappa^{2} \\
\hline \kappa & 0 & & \\
\kappa^{2} & -1 & 1 &
\end{array}
$$$$
\kappa^{3} \quad 4 \begin{array}{lll}
4 & -5 & 5
\end{array}
$$

In particular, we note that the $\kappa$-weights of the remaining values of $T^{*}$ are a claimed. 


\section{Groups}

We recall some standard notation. Let $G$ be a maximal class $p$-group of order $p^{n}$ and $\gamma_{i}(G)$ denote the terms of the lower central series. Let $P_{i}=P_{i}(G)=\gamma_{i}(G)$ for $2 \leq i \leq n$ and let $P_{1}=P_{1}(G)$ be the centralizer in $G$ of $P_{2} / P_{4}$, and $P_{0}=G$. Then the $P_{i}$ form a chief series of $G$.

Let $s$ and $s_{1}$ denote elements of $G$ with $s \in G \backslash P_{1}$ and $s_{1} \in P_{1} \backslash P_{2}$ and define $s_{i}=\left[s_{i-1}, s\right]$ for $2 \leq i \leq n$. If $G$ has positive degree of commutativity, then Lemma 3.2 .4 of [5] says that $P_{i}=\left\langle s_{i}\right\rangle P_{i+1}$, for $1 \leq i \leq n$. In this case it follows that every element of $G$ has a unique representation of the form $s^{e_{0}} s_{1}^{e_{1}} s_{2}^{e_{2}} \cdots s_{n-1}^{e_{n}-1}$ where $0 \leq e_{i}<p$.

Following [5, p.157], let $G$ be a 5-group of maximal class of order $5^{n}$ with positive degree of commutativity. Suppose that $P_{1}$ is class 2 and let $m$ be such that $P_{1}^{\prime}=P_{m}$. Then $P_{1} / P_{m}$ and $P_{m}$ are abelian. By Lemma 8.2.1 of [5], we have $\mathcal{O}$-module isomorphisms $f_{G}: \mathcal{O} / \mathfrak{p}^{m-1} \rightarrow P_{1} / P_{m}$ and $g_{G}: \mathcal{O} / \mathfrak{p}^{n-m} \rightarrow P_{m}$ given by

$$
\left(\mathfrak{p}^{m-1}+a_{0}+a_{1} \kappa+\cdots+a_{m-2} \kappa^{m-2}\right) f_{G}=P_{m} s_{1}^{a_{0}} s_{2}^{a_{1}} \ldots s_{m-1}^{a_{m-2}}
$$$$
\left(\mathfrak{p}^{n-m}+a_{0}+a_{1} \kappa+\cdots+a_{n-m-1} \kappa^{n-m-1}\right) g_{G}=s_{m}^{a_{0}} s_{m+1}^{a_{1}} \ldots s_{n-1}^{a_{n-m-1}} \text {. }
$$
Define

Then commutation in $P_{1}$ induces a homomorphism $\eta_{G}$ from $\wedge^{2}\left(P_{1} / P_{m}\right) \rightarrow P_{m}$.

$$
\alpha_{G}=\left(f_{G} \wedge f_{G}\right) \eta_{G} g_{G}^{-1}: \mathcal{O} / \mathfrak{p}^{m-1} \wedge \mathcal{O} / \mathfrak{p}^{m-1} \rightarrow \mathcal{O} / \mathfrak{p}^{n-m} .
$$

Note that $\alpha_{G}$ is built out of commutation and, in particular, if $\zeta=\left(\kappa^{i} \wedge \kappa^{j}\right) \alpha_{G}$, then $\zeta g_{G}$ is just the commutator $\left[s_{i+1}, s_{j+1}\right]$.

The next theorem provicles some details about this homomorphism $\alpha_{G}$. In order to describe $\alpha_{G}$ it is useful to note that the homomorphisms $T_{1}$ and $T^{*}$ map from $\mathcal{O} \wedge \mathcal{O}$ to $\mathcal{O} / \mathfrak{p}^{n-m}$. Now, $\mathcal{O} / \mathfrak{p}^{m-1} \wedge \mathcal{O} / \mathfrak{p}^{m-1} \cong(\mathcal{O} \wedge \mathcal{O}) / I$ for some $C_{5}$-submodule $I$. In [4, Section 7] it is shown that this $I$ is in the kernel of each of $T_{1}$ and $T^{*}$ and so each induces a homomorphism from $\mathcal{O} / \mathfrak{p}^{m-1} \wedge \mathcal{O} / \mathfrak{p}^{m-1}$ to $\mathcal{O} / \mathfrak{p}^{n-m}$.

THEOREM 3.1. Let $G$ be a group of maximal class of order $5^{n}$ with $P_{1}^{\prime}=P_{m}$ central in $P_{1}$ where $n \geq m \geq 4$. We assume $G$ has positive degree of commutativity (this only rules out a few groups of order $5^{6}$ ).

If $P_{1}$ is not abelian, then $G$ corresponds to a homomorphism $\alpha_{G}$ induced by $a T_{1}+b T^{*}$ where $a \in \mathcal{O}, 0 \leq b \leq 4$, and if $a \in \mathfrak{p}$, then $n=m+1$ and $b \neq 0$. Also one of the following holds:

(1) $m \equiv 1 \bmod 4$ and $2 m \geq n+1$,

(2) $m>4, m \neq 1$ mod 4 and $2 m \geq n+2$,

(3) $m=4, n=7$ and $b \equiv a \bmod p$

(4) $m=4, n=5$ or 6 and $b=0$.

Proof. This is part of Theorem 7.6 of [4].

We now wish to compute the pattern of commutators in $P_{1}$ for some special cases of $\alpha_{G}$.

REMARK 3.2. Any value of $k$-weight $k$ is mapped by $g_{G}$ to an element of the form

$$
s_{m+k-1}^{a_{m+k-1}} s_{m+k}^{a_{m+k}} \ldots s_{n-1}^{a_{n-m-1}}
$$

LEMMA 3.3. If $G$ is a group with $\alpha_{G}$ induced by $T_{1}$, then $\left|P_{i}^{\prime}\right|=p^{2}\left|P_{i+1}^{\prime}\right|$ unless $\left|P_{i+1}^{\prime}\right|=1$. If $\left|P_{i+1}^{\prime}\right|=1$ then $\left|P_{i}^{\prime}\right| \leq p^{2}$.

Proof. Fix $r$ and consider $P_{r}^{\prime}$. This subgroup is generated by the $g_{G}$-images of $\left\{\left(\kappa^{i} \wedge \kappa^{j}\right) T_{1}\right\}$ where $m-1>i>j \geq r-1$. By Corollary 2.4 these $T_{1}$ values include items of $\kappa$-weight $2 r-1,2 r, 2 r+1, \ldots, 2 m-5$. We want to know that $2 m-5 \geq n-m$ or, equivalently, $3 m \geq n+5$. By Theorem $3.12 m \geq n+1$ and since $m \geq 4$, we have $3 m \geq n+5$. So, we can say that the $T_{1}$ values above include items of $\kappa$-weight $2 r-1,2 r, 2 r+1, \ldots, n-m$.

Based on these $\kappa$-weights, the $g_{G}$-images of these values will include elements of $G$ with leading terms $s_{m+2 r-2}, s_{m+2 r-1}, \ldots, s_{n-1}$. It follows that $P_{r}^{\prime}=P_{m+2 r-2}(G)$. From this formula, the stated conditions on $\left|P_{i}^{\prime}\right|$ are immediate.

LEMMA 3.4. If $G$ is a group with $\alpha_{G}$ induced by $T^{*}$, then $\left|P_{1}^{\prime}\right|=\left|P_{2}^{\prime}\right|=5$, and $\left|P_{i}^{\prime}\right|=1$ for $i \geq 3$

Proof. By Corollary 2.5, the only non-trivial values of $\left(\kappa^{i} \wedge \kappa^{j}\right) T^{*}$ have $\kappa$ weight $n-m$ and so $P_{1}^{\prime}=P_{2}^{\prime}=P_{n-1}(G)$.

Combining these, we have

THEOREM 3.5. The possible values of the sequence $\left|P_{1}^{\prime}\right|,\left|P_{2}^{\prime}\right|, \ldots$ for a maximal class 5-group with $P_{1}$ of nilpotence class 2 are:

$$
\begin{aligned}
& p^{2 k-1}, p^{2 k-3}, \ldots, p, 1,1, \ldots \\
& p^{2 k}, p^{2 k-2}, \ldots, p^{2}, 1,1, \ldots \\
& p^{2 k}, p^{2 k-2}, \ldots, p^{2}, p, 1,1, \ldots \text { for } k \geq 1
\end{aligned}
$$$$
\text { or }
$$

$p, p, 1,1, \ldots$

Proof. We first note that the computations above often assume that $G$ has positive degree of commutativity. This is guaranteed if $|G|>5^{6}$. Using the SmallGroups database [1], we check the properties of small maximal class 5-groups with $P_{1}$ having class 2 . The 6 groups of order $5^{5}$ and 25 groups of order $5^{6}$ have sequences $\left|P_{1}^{\prime}\right|,\left|P_{2}^{\prime}\right|, \ldots$ equal to $(5,1, \ldots),(5,5,1, \ldots)$, or $(25,1, \ldots)$.

Now we can assume that $G$ has positive degree of commutativity and so $\alpha_{G}=$ $a T_{1}+b T^{*}$ as above.

First we consider $b=0$. By Lemma 3.3, the desired result holds if $a=1$ and, similary, if the $\kappa$-weight of $a$ is 1 . However, if the $\kappa$-weight of $a$ is greater than 1 then the $\kappa$-weight of $\left(\kappa^{i} \wedge \kappa^{j}\right) a T_{1}$ is uniformly larger than $\left(\kappa^{i} \wedge \kappa^{j}\right) T_{1}$ and so the indices $\left|P^{\prime}: P^{\prime}\right|$ will not change unless the subgroups in question become trivial. Consequently, the sequence $\left|P_{1}^{\prime}\right|,\left|P_{2}^{\prime}\right|, \ldots$ will still fall into one of the patterns given, Consequently, the sequence $\left|P_{1}^{\prime}\right|,\left|P_{2}^{\prime}\right|, \ldots$ will still fall into
but the values will be smaller and will reach 1 sooner.

Now if $b>0$, the addition of $\left(\kappa^{i} \wedge \kappa^{j}\right) b T^{*}$ will affect at most $\left|P_{1}^{\prime}\right|$ and $\left|P_{2}^{\prime}\right|$. Furthermore, since we are only introducing values of $\kappa$-weight $n-m$, the orders of the commutator subgroups will only be affected if they are trivial. That is, sequences of the form $25,1, \ldots$ and $5,1, \ldots$ will become $25,5,1, \ldots$ and $5,5,1, \ldots$ each of which are in the stated list.

\section{Character Degrees}

If $G$ is normally monomial, the sequence $\left|P_{1}^{\prime}\right|,\left|P_{2}^{\prime}\right|, \ldots$ is sufficient to compute the character degrees of $G$ as follows. 
LEMMA 4.1. Let $G$ be a normally monomial p-group of maximal class. Then $\operatorname{cd}(G)-1=\left\{\left|G: P_{i+1}\right|, 0 \leq i<n\right.$ such that $\left.P_{i}^{\prime}>P_{i+1}^{\prime}\right\}$

Proof. This result is found in the proof of Corollary 2.6 in [3]

This allows us to classify the possible character degrees when $P_{1}$ has class 1 or

THEOREM 4.2. Let $G$ be a normally monomial, maximal class 5-group with $P_{1}(G)$ at most class 2. Then $\operatorname{cd}(G)$ is either $\{1,5,125\}$ or the set of all powers of 5 up to some limit, $\left\{1,5,25, \ldots, 5^{k}\right\}, k \geq 1$.

PRoof. If $P_{1}$ is abelian, then $G$ has an abelian group of index $p$ and so the possible character degrees are 1 and $p$.

Otherwise, $P_{1}$ has class 2 and we can apply Theorem 3.5 to deduce possible values for $\left|P_{1}^{\prime}\right|,\left|P_{2}^{\prime}\right|, \ldots$. In particular, the non-trivial orders if $P_{i}^{\prime}$ strictly decrease in every case except $(5,5,1, \ldots)$. For these strictly decreasing sequences, Lemma 4.1 implies that the character degrees of $G$ will form a full set of powers of 5 up to some limit, $\left\{1,5,25, \ldots, 5^{k}\right\}, k \geq 1$.

On the other hand a commutator subgroup pattern of $5,5,1, \ldots$ implies

$$
\operatorname{cd}(G)=\left\{1,\left|G: P_{1}\right|,\left|G: P_{3}\right|\right\}=\{1,5,125\}
$$

Now, a result of Mann's will allow us to lift this character degree information to any normally monomial, maximal class 5-group (regardless of class of $P_{1}(G)$ ).

LEMMA 4.3. Let $G$ be a normally monomial p-group satisfying $\left|G: G^{\prime}\right|=p^{2}$, and let $\operatorname{cd}(G)=\left\{1, p, p^{r_{3}}, \ldots, p^{r_{k}}\right\}$. If $M$ is a maximal subgroup of $G$, then $\operatorname{cd}(M)$ consists of 1 , possibly $p$, and the numbers $p^{r_{i}-1}$.

Proof. This is one case of Corollary 13 of [6]

Treoram 4.4. Let $G$ be a normally monomial, $m$ $\operatorname{cd}(G)$ is either $\left\{1,5,25,5^{4}\right\}$, the set $\left\{1,5,25, \ldots, 5^{k}\right\}$ with $k \geq 1$ of all powers of 5 up to some limit, or either of those two forms with degree 25 removed.

Proof. Let $G$ be any normally monomial maximal class 5-group, and let $M$ be a maximal subgroup $G$ not equal to $P_{1}$. Then, by [6], $M$ is normally monomial and maximal class. Furthermore, by Corollary 3.4 .12 of [5] (with $p=5), P_{1}(M)=$ $P_{2}(G)$ has class at most 2. Thus, by the previous section, $\operatorname{cd}(M)$ is constrained. Now, the preceding lemma shows that $\operatorname{cd}(G)$ is closely determined by $\operatorname{cd}(M)$ and so we deduce that $\operatorname{cd}(G)$ must be one the forms listed.

\section{Future Directions}

As mentioned in the Introduction, I only know of maximal class 5-groups which have character degrees $\left\{1,5,5^{3}\right\}$ or $\left\{1,5,25, \ldots, 5^{k}\right\}$. Thus the current result, while nice, is probably not the end of the story, even for 5-groups.

A natural question is to ask what happens for $p=7,11, \ldots$. It seems likely that the character degrees of maximal class 7-groups will have all of the 5-group patterns (i.e. $\left\{1,7,7^{3}\right\}$ and $\left\{1,7,49, \ldots, 7^{k}\right\}$ ) and it appears from very prelininary computations that some other patterns of powers of 7 show up as well. I conjecture that (as with $p=5$ ) there are sets of powers of 7 containing 1 and 7 that don't and (as maximal class 7-group.

The thechniques of this paper to $p=7$ (and There are a few difficulties in applying the techniques of this paper combinations of higher). For $p=7$, the homomorphisms $\alpha_{G}$ which arise are linear combinations ore $T_{1}, T^{*}$ and another map $T_{2}$. Linear combinations of $T_{1}$ and $T_{2}$ seem to have more opportunities for interaction which will probably make the case analysis harder. Similarly the fruct $\mathcal{O} \wedge \mathcal{O}$ is more complicated. It remains true that maximal Similarly, the structure of 0 is most 2 , but, by $p=11$, groups of derived length 3 and more begin to appear.

\section{References}

1. H.U. Besche, B. Eick, and E. A. O'Brien, A millennium project: Constructing small groups, Internat. J. Algebra Comput., 12 (2002), 623-644.

Magma algebra system I. The user Language, J. Symbolic Comput., 24(1997), 235-265.

J. Keller, D. Ragan, and G.T. Tims, On the Taketa bound for normally monomial p-groups of maximal class, J. Algebra, 277 (2004), 675-688.

C.R. Leedham-Green and S. McKay, On p-groups of maximal class III, Quart. J. Math Oxford (2), 29 (1978), 281-299.

5. C. Leedham-Gro

27 Oxford University Press, 2002.

A. Mann, Normally monomial p-groups, J. Algebra, 300 (2006), 2-9.

Dept. OF Math, Stat., and Comp. Sci., Marouette Univ., Milwaukee, WI 53201-188 E-mail address: mikes@mscs,mu.edu 